\title{
Poly(alkylene 2,5-furanoate)s thin films: morphology, crystallinity and nanomechanical properties
}

Beatriz Robles-Hernández ${ }^{1,2}$, Michelina Soccio ${ }^{3} *$, Iker Castrillo $^{2}$, Giulia Guidotti ${ }^{3}$, Nadia Lotti $^{3}$, Ángel Alegría ${ }^{1,2}$, Daniel E. Martínez-Tong ${ }^{1,2, *}$

${ }^{1}$ Departamento de Física de Materiales, University of the Basque Country (UPV/EHU). P. Manuel de Lardizábal 3, E-20018 San Sebastián - Spain.

${ }^{2}$ Centro de Física de Materiales (CSIC -UPV/EHU). P. Manuel de Lardizábal 5, E-20018 San Sebastián - Spain

${ }^{3}$ Civil, Chemical, Environmental and Materials Engineering Dept., University of Bologna, Via Terracini 28, 40131, Bologna - Italy 


\section{ABSTRACT}

Poly(alkylene 2,5-furanoate)s are considered as the most attractive and interesting alternatives to replace oil-based terephthalic polymers. These furan-based polyesters can be synthesized using fully bio-based synthetic strategies, allowing to reduce the environmental impact of plastics. At the same time, these polymers have shown outstanding thermal, mechanical and gas-barrier properties. All these results envisage their industrial use in the near future. Now, considering the downscaling of the products' size towards the nanometer scale, we present a study of the morphology and nanomechanical properties of poly(alkylene 2,5-furanoate) thin films. Using Atomic Force Microscopy, we report the development of nanostructures upon crystallization, following different thermal treatments, for thin films with thicknesses below $200 \mathrm{~nm}$. Moreover, we studied the impact of crystal growth in the nanomechanical properties of these materials. We found that the polymer thin films preserve their excellent mechanical response even in the confined geometry, as proved by the Young's moduli values close to the GPa, accompanied by high surface stiffness, and low indentation depths. The poly(alkylene 2,5-furanoate) thin films were found to have nanomechanical properties comparable to those of the oil-based poly(ethylene terephthalate), a further evidence that in the future they could replace traditional polymers in several applications.

KEYWORDS: poly(alkylene 2,5-furanoate)s, thin films, atomic force microscopy, polymer nanostructures, polymer crystallization, nanomechanical properties. 


\section{Introduction}

In recent years, there has been an increasing interest towards the study of furan-based polymer materials. Reports on the synthesis and properties of such polymers are now abundant in the literature, most of them dealing with poly(ethylene 2,5-furanoate) [1-4] and poly(propylene 2,5- furanoate) (PPF) [5-7]. More recently, poly(alkylene 2,5-furanoate)s with longer glycolic subunits have also attracted the attention of researchers [8-11]. In most of these works, the research groups have emphasized two aspects. First, the poly(alkylene 2,5furanoate)s can be obtained using renewable sources. Second, the obtained materials present excellent mechanical, thermal, and gas-barrier properties. In fact, all the so-far published articles indicate that poly(alkylene 2,5-furanoate)s have better properties that their terephthalic counterparts. If both of these aspects are considered, it is not surprising that they are considered the most credible alter ego of terephthalic polyesters. It is also widely believed that their entry on the market will entail the environmental impact of the plastics industry.

As fairly discussed in the literature, several tools can be used to tune the polymer final properties in such a way to fit the requirements for a specific functional application. Among these tools, we can cite the modification of the chemical structure of the repeating units, synthesis of copolymers, physical and reactive polymer blending, and last but not least, the preparation of "ad hoc" composites and nanocomposites. As to the first tool, literature reports several papers dealing with furan-based homopolyesters, characterized by different chemical structure. Most of the papers describe the effect of glycol sub-unit length on the polymers' properties $[6,9,11-22]$. Regarding other tools, the development of furan-based materials for functional applications, and the tuning of their physical properties, has been also achieved by copolymerization [23], nanocomposites preparation [24, 25], and by the development of surface nanostructures [26]. For example, in the latter case, a work recently carried out by 
Soccio and collaborators reported on the fabrication of wrinkled PPF surfaces by laser irradiations [26]. In this investigation, nanometric gratings were prepared on bulk PPF freestanding films. Via force spectroscopy measurements, the authors demonstrated that by nanostructuring the PPF surface, it was possible to improve its mechanical properties without compromising its chemical structure, contrary to previous observations in terephthalic-based counterparts. This was quantified by an increase in the Young's modulus and surface stiffness.

All the aforementioned works dealt with bulk furan-based polymers. In order to push forward the research in these materials, in our present work, we report the preparation, and the study of crystallization and nanomechanical properties of poly(alkylene 2,5-furanoate)s in thin film geometry. Our study is motivated by the continuous miniaturization of technological devices and components, where the polymer industry has been proven to be fundamental. Specifically, a recent review about polymer thin films spotlighted their use in chips, flat panel displays, photovoltaic devices, and coatings [27]. Moreover, polymer thin films have been a subject of academic debates for over the past 20 years [28-31]. In the particular case of semicrystalline polymers, the confinement imposed by the thin film geometry, and the polymer-substrate adsorption effects, resulted in differences in the crystallization rate [32, 33], crystalline morphology [34-36], thermal transitions and polymers' molecular dynamics [32, 37]. In turn, preparing poly(alkylene 2,5-furanoate)s can be also a way to tune their physical properties, at nanoscale levels.

In the present work, we focused on poly(alkylene 2,5-furanoate)s characterized by different glycolic subunits (the number of carbon atoms ranged from 4 to 6 ). In all cases, we were able to prepare thin films, with thicknesses below $150 \mathrm{~nm}$. We explored the crystalline nanostructure growth and morphology, after different thermal treatments, by using Atomic Force Microscopy 
(AFM). Also, in particular cases of interest, we explored the thickness dependence on the crystalline nanostructure morphology. Finally, we present a full nanomechanical study on these materials. Using AFM-based techniques, we were able to explore the nanomechanical contrast induced by the crystalline structures, and the impact of the nanometric features in the surface mechanical response.

\section{Experimental Section}

\subsection{Materials and methods}

Poly(butylene 2,5-furanoate) (PBF), poly(pentamethylene 2,5-furanoate) (PPeF), and poly(hexamethylene 2,5-furanoate) (PHF) have been used for preparing thin films. The chemical structure of these polymers is presented in Scheme 1 with the subscript " $n$ " indicating the number of methylene groups in the glycol subunit; $\mathrm{n}$ is 4 for PBF, 5 for PPeF and 6 for PHF. All three samples were synthesized as previously described [8, 9, 38].<smiles>COC(C)(C)COC(=O)c1ccc(C(C)=O)o1</smiles>

Scheme 1. Chemical structure of the investigated poly(alkylene 2,5-furanoate)s; $n$ is 4,5 and 6 for PBF, PPeF and PHF, respectively.

The thermal characterization of the bulk samples can be found in recent literature reports $[9$, 38, 39], with the magnitudes derived from such calorimetric studies summarized in Table 1. The crystallization behavior of the poly(alkylene 2,5-furanoate)s has a clear dependence on the number of methylene groups on the repeating unit. In particular, an odd-even effect was reported, being the polymers containing an even number of $-\mathrm{CH}_{2}$ - in glycol sub-units, i.e., $\mathrm{PBF}$ 
and PHF, faster in crystallizing [11]. However, these two samples show very different crystallization kinetics: PHF crystallizes readily, almost independently from thermal history, while PBF presents distinct structural states depending on thermal treatment [39]. This behavior allows tuning the development of crystalline structures on PBF samples by playing with thermal treatments, as presented in the present manuscript. Finally, the PPeF sample, being the only one with a C-odd-numbered glycolic subunit, does not crystallize in "regular" laboratory times $[9,11,40]$. Concerning the glass transition temperature $\left(T_{\mathrm{g}}\right)$, according to recent studies, there is a decrease in $T_{\mathrm{g}}$ as the length of the alkyl chain increases $[11,38]$. In this way, by increasing the length of the aliphatic subunit, the system changes from a glassy material (PBF) to a rubbery one (PPeF and PHF) at room temperature.

Table 1. Thermal characterization results for the studied poly(alkylene 2,5-furanoate)s.

\begin{tabular}{c|ccccccc} 
Sample & $\begin{array}{c}T_{\mathrm{g}} \\
\left({ }^{\circ} \mathrm{C}\right)\end{array}$ & $\begin{array}{c}\Delta C_{\mathrm{p}} \\
\left(\mathrm{J} / \mathrm{g}^{\mathrm{o}} \mathrm{C}\right)\end{array}$ & $\begin{array}{c}T_{\mathrm{cc}} \\
\left({ }^{\circ} \mathrm{C}\right)\end{array}$ & $\begin{array}{c}\Delta H_{\mathrm{cc}} \\
(\mathrm{J} / \mathrm{g})\end{array}$ & $\begin{array}{c}T_{\mathrm{m}} \\
\left({ }^{\circ} \mathrm{C}\right)\end{array}$ & $\begin{array}{c}\Delta H_{\mathrm{m}} \\
(\mathrm{J} / \mathrm{g})\end{array}$ & $R e f$. \\
\hline$P H F$ & $13 \pm 1$ & $0.205 \pm 0.005$ & -- & -- & $144 \pm 1$ & $40 \pm 1$ & {$[38]$} \\
$P P e F$ & $13 \pm 1$ & $0.394 \pm 0.005$ & -- & -- & $42 \pm 1$ & $23 \pm 1$ & {$[9,40]$} \\
$P B F-f c$ & $35 \pm 1$ & $0.22 \pm 0.01$ & $67 \pm 1$ & $36 \pm 1$ & $157 \pm 1$ & $37 \pm 1$ & \\
$P B F-s c$ & $43 \pm 3$ & $0.14 \pm 0.03$ & -- & -- & $161 \pm 1$ & $37 \pm 1$ &
\end{tabular}

$f c$ : fast cooled, sc: slow cooled, according to Ref [39].

\subsection{Film Preparation and Atomic Force Microscopy (AFM) measurements.}

Poly(alkylene 2,5-furanoate) thin films were prepared by spin coating polymer solutions (3000 rpm, $2 \mathrm{~min}$ ) onto silicon oxide wafers (TedPella, ref: 16008). Depending on the polymer, different solvents and conditions were used, as summarized in Table 2. Prior coating, the substrates were cleaned in acetone and dried under $\mathrm{N}_{2}$ flow. Following previous reports 
[41], after the spin coating, all samples were left in a high vacuum $\left(<10^{-5}\right.$ torr $)$ chamber overnight at room temperature, to evaporate the possible residual solvent.

Based on the reported DSC results (Table 1), we designed suitable thermal protocols to allow the formation of crystalline nanostructures. First, in a so-called "fast-cooled" protocol, the samples were heated up to $180{ }^{\circ} \mathrm{C}$, at a $15 \mathrm{~K} \cdot \mathrm{min}^{-1}$ rate. The final temperature was held for 1 min and immediately afterwards the thin films were placed onto a cold plate (temperature $\approx$ $\left.25^{\circ} \mathrm{C}\right)$. Second, the so-called "slow-cooled" samples were treated by heating up the thin films up to $180{ }^{\circ} \mathrm{C}$, at $15 \mathrm{~K} \cdot \mathrm{min}^{-1}$, and then cooling them down to room temperature at a controlled rate of $3 \mathrm{~K} \cdot \mathrm{min}^{-1}$. All thermal treatments were performed using a Linkam THMS600 stage connected to a T95-LinkPad System controller.

Table 2. Experimental conditions used for preparing poly(alkylene 2,5-furanoate) thin films.

\begin{tabular}{l|ccccc} 
Sample & Solvent & $\begin{array}{c}T \\
\left({ }^{\circ} \mathrm{C}\right)\end{array}$ & $\begin{array}{c}\text { Stirring } \\
\text { time } \\
(\text { hours })\end{array}$ & $\begin{array}{c}\text { Solution } \\
\text { concentration } \\
\left(\mathrm{g} \mathrm{L}^{-1}\right)\end{array}$ & $\begin{array}{c}\text { Thickness } \\
(\mathrm{h}) \\
(\mathrm{nm})\end{array}$ \\
\hline PHF & Chloroform & 50 & 2 & 20 & 190 \\
$P P e F$ & Chloroform & 25 & 2 & 20 & 150 \\
$P B F$ & $\begin{array}{c}\text { Chloroform:Hexalfuoro-2-propanol } \\
(80: 20 \mathrm{v} / \mathrm{v})\end{array}$ & 25 & 24 & 27 & 110
\end{tabular}

A Multimode AFM equipped with a NanoScope V controller (Bruker), running NanoScope 8.15 (Build R3Sr8.103795), was used for surface imaging and nanomechanical studies. The topographical studies were carried out in tapping mode, using Tap150Al-G silicon nitride probes (BudgetSensors), with a mean resonant frequency $\left(f_{0}\right) \sim 120 \mathrm{kHz}$. The thickness $(h)$ of the samples was determined by performing AFM images next to a scratch, drawn for this 
purpose, which results are summarized in Table 2. The AFM topography images were processed using the Gwyddion 2.54 software (http://gwyddion.net/) [42].

Lately, there has been a continuous increase in the use of high-resolution nanomechanical measurements to study polymer materials [43-49]. In our work, the nanomechanical studies were performed combining the PeakForce-Quantitative Nanomechanical Mapping (PF-QNM) protocol by Bruker, with force spectroscopy measurements. For these experiments, we used Tap300Al-G silicon nitride probes (BudgetSensors), with a cantilever spring constant in the $22-27 \mathrm{~N} \cdot \mathrm{m}^{-1}$ range, as determined by Sader's method [50]. The tip radius was found using a polystyrene thin film, as described below. Using PF-QNM, we were able to map the samples' topography while obtaining mechanical contrast information, simultaneously. The sinusoidal modulation frequency $\left(f_{\mathrm{m}}\right)$ of the PF-QNM experiments was selected as $2 \mathrm{kHz}$, and the peak force parameter was kept constant and equal to $30 \mathrm{nN}$. The deflection sensitivity of the AFM probes was calculated using a sapphire standard (Bruker) leading to values of $40 \mathrm{~nm} / \mathrm{V}( \pm 10 \%$ error) in both linear ramps and during PF-QNM sinusoidal modulation, under the same force conditions. The PF-QNM nanomechanical maps were evaluated using the NanoScope 1.90 software (Bruker).

Based on the PF-QNM maps, and using the same calibration, we performed force spectroscopy measurements on selected regions of the thin films. For these measurements, we applied a linear ramp of $200 \mathrm{~nm}$ length in the $Z$ direction of the piezo scanner (vertical movement). The interaction between the sample and the AFM probe, during ramping, allowed recording a force vs. tip-sample separation curve, as presented in Figure S1 (Supporting Information) for a polystyrene thin film (PS, thickness $=150 \mathrm{~nm}$ ). For all the investigated samples, we used a trigger threshold of $1.2 \mathrm{~nm}$, corresponding to $\sim 30 \mathrm{nN}$ of maximum applied 
force, the ramp rate was $1 \mathrm{~Hz}$, and we collected 9728 data points. From the approach curve (blue colored dots in Figure S1) we calculated the sample stiffness, as $\Delta F / \Delta \delta$, in the $50-90$ $\%$ range of the total applied force. In addition, from this curve, we calculated the indentation depth $\left(\delta_{\mathrm{i}}\right)$ as the $Z$-separation distance between the point of zero force, after van der Waals interactions took place, and the maximum load. From the retraction curve (orange colored dots in Figure S1) we calculated the adhesion force $\left(F_{\mathrm{adh}}\right)$ as the minimum force point while the tip withdraws. Finally, by fitting the retraction curve we obtained the sample's Young's modulus $\left(E_{\mathrm{Y}}\right)$, using the Hertz model of contact mechanics:[51]

$$
F=\frac{4}{3} \frac{E_{\mathrm{Y}}}{\left(1-v^{2}\right)} \sqrt{R_{\mathrm{tip}}} \delta_{\mathrm{i}}^{3 / 2}
$$

where $v$ is the Poisson's ratio, set as 0.3 . In the specific case of the PS, we used the force spectroscopy results for obtaining the tip radius. In this case, the fitting of eqn. (1) was carried out fixing $E_{\mathrm{Y}}(\mathrm{PS})=3.3 \mathrm{GPa}$ and leaving the $R_{\text {tip }}$ parameter free. By this process, we obtained a tip radius of $45 \mathrm{~nm}$. This value was also used for obtaining the PF-QNM maps.

\section{Results \& Discussion}

\subsection{Poly(alkylene 2,5-furanoate)s thin films: morphology and crystal growth}

Figure 1 shows $2 \times 2 \mu \mathrm{m}$ AFM height images of the PHF, PPeF and PBF thin films. The left column in Figure 1 shows the as-casted samples' surface topography. Here, the poly(alkylene 2,5-furanoate)s with a C-even-numbered glycolic subunit showed a nanostructured surface with distinct geometrical characteristics depending on the sample, while the PPeF, having a C-odd-numbered glycolic subunit, showed a flat surface regardless of the thermal treatment. Going into detail, the surface of the PHF thin film (Figure 1 a)) was composed by a sort of small aggregates with a mean lateral size of $\sim 20 \mathrm{~nm}$; the PPeF thin film presented a continuous and flat surface without any features (Figure $1 \mathbf{b}$ )); and the PBF sample showed a nanostructured surface composed by circular inclusions, with a mean diameter $\sim 150$ 
$\mathrm{nm}$ and a mean depth $\sim 6 \mathrm{~nm}$ (Figure $1 \mathrm{c})$ ). Please, beware that the PBF surface discontinuities reached a depth of only about $5 \%$ of the total sample thickness, allowing us to conclude that this thin film did not undergo a dewetting process. In Figure S2, we present zoom-in AFM topography images of PHF and PBF, to detail their different nanometric features.
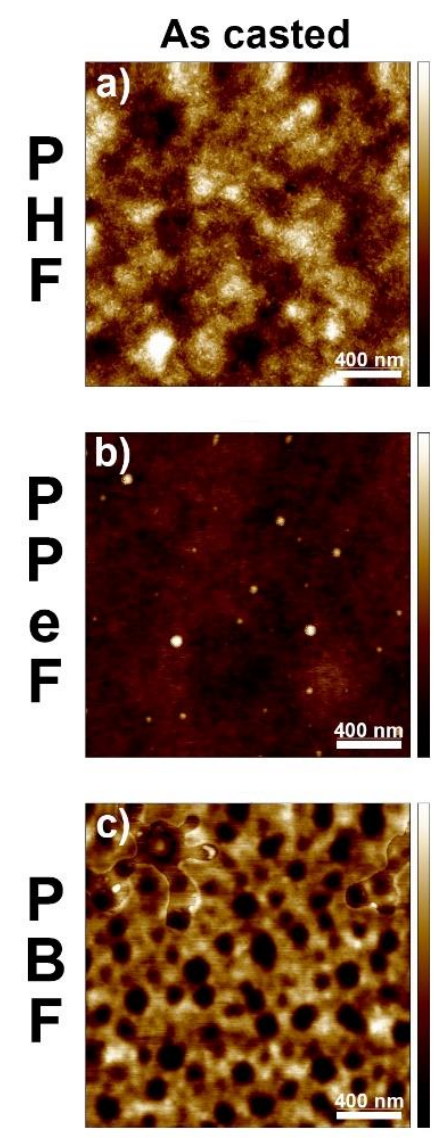

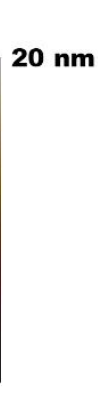

$5 \mathbf{~ n m}$

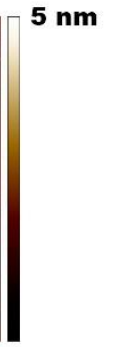

$10 \mathrm{~nm}$
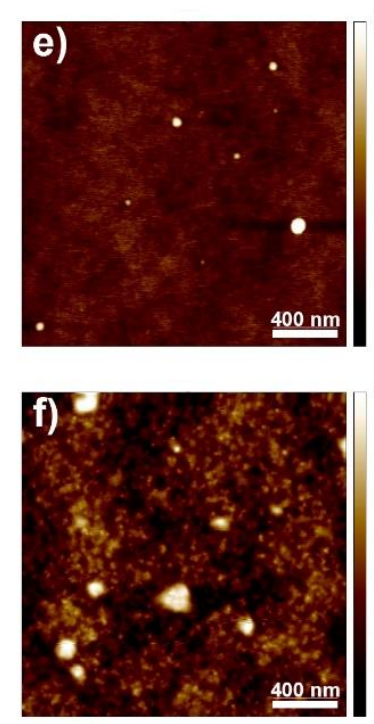

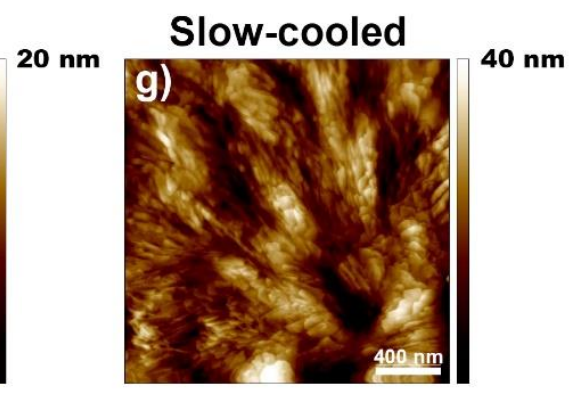

5 nm

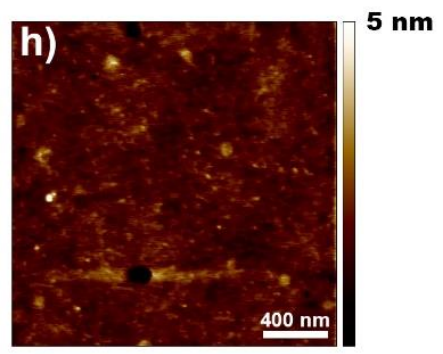

$10 \mathrm{~nm}$

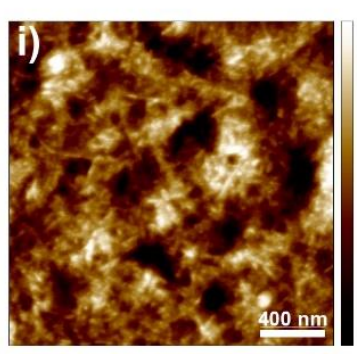

$20 \mathrm{~nm}$

Figure 1. AFM height images of the studied poly(alkylene 2,5-furanoate) thin films subjected to different thermal treatments: a)-c) as-casted films, d)-f) fast-cooled films, and g)-i) slowcooled films. As can be observed, the PPeF does not show signatures of crystallization after slow-cooling from the melt.

To provide further information on the topography of the films, we calculated the mean surface roughness $\left(R_{\mathrm{a}}\right)$ of the thin films as:

$$
R_{a}=\frac{1}{N} \sum_{j=1}^{N}\left|Z_{j}-Z_{0}\right|
$$


where $N$ is the total number of pixels, and $\left|Z_{j}-Z_{0}\right|$ the average height value at the $j$-th pixel of the image, from the mean plane. In general, amorphous polymer thin films show $R_{\mathrm{a}}$ values well below $1 \mathrm{~nm}$ [52], while semicrystalline polymer thin films usually present $R_{\mathrm{a}}$ values around and above $1 \mathrm{~nm}[53,54]$. As reported in

Table 3, the as-casted PHF surface had a $R_{\mathrm{a}}$ value of $2.7 \mathrm{~nm}$ that, in combination with its fast crystallization kinetics $[10,38]$, seems to indicate that the observed nanostructures are composed by small polymer crystals. In the case of the PPeF sample, we quantified a very low $R_{\mathrm{a}}$ value of $\sim 0.3 \mathrm{~nm}$, which indicates that the as-casted thin film would be fully amorphous, in line with the bulk DSC reports $[9,11]$. Finally, in the case of the as-casted PBF film, the $R_{\mathrm{a}}$ value was $1.6 \mathrm{~nm}$. Then, the roughness value obtained for the PBF surface indicates the presence of crystalline structures, as for PHF. It is worth noting that we calculated the roughness of this PBF thin film considering the whole image, including the holes developed during the deposition process. Then, even considering the discontinuities, $R_{\mathrm{a}}$ of the as-casted PBF thin film was $\sim 40 \%$ lower than that found for PHF. The nature of the PBF circular inclusions is not obvious; however, a possible explanation of this result could be related to the use of a mixture of solvents to prepare PBF films. Chloroform and hexafluoro-2-propanol, although miscible, present different physicochemical properties that can lead to differences in the evaporation process during spin coating. In fact, in PPeF and PHF as-casted thin films, for which only chloroform was used as solvent, no inclusions were observed.

Table 3. Mean surface roughness values $\left(R_{\mathrm{a}}\right)$ of the poly(alkylene 2,5-furanoate) thin films, prepared following different thermal treatments.

\begin{tabular}{l|cccc}
\multicolumn{5}{c}{$R_{\mathrm{a}}(\mathrm{nm})$} \\
\hline Sample & As-casted & Fast-cooled & Slow-cooled & Aged* $^{*}$ \\
\hline PHF $(h=150 \mathrm{~nm})$ & 2.68 & 2.33 & 5.33 & 2.37
\end{tabular}




\begin{tabular}{l|cccr}
$P P e F(h=150 \mathrm{~nm})$ & 0.27 & 0.22 & 0.29 & 8.80 \\
$P B F(h=112 \mathrm{~nm})$ & 1.56 & 1.03 & 2.71 & 1.34 \\
\multicolumn{5}{r}{ *This mean surface roughness value corresponds to the 6 months aged thin films. }
\end{tabular}

It is reasonable consider that the as-casted polymer films have undergone a quenching treatment during spin coating, the solvent evaporation time being very small (usually $<2$ seconds). Nevertheless, we cannot ignore literature reports indicating that spin casted polymer thin films are in a metastable state due to the stress produced during spinning [55]. Then, in order to normalize the thermal history and explore further nanostructure formation, the poly(alkylene 2,5-furanoate) thin films were subjected to different thermal protocols (see Experimental Section). This procedure would also discard the influence of the solvent used in the film preparation, as reported previously for other polymer thin films $[56,57]$. The center column in Figure 1 shows the AFM height images for fast-cooled thin films. After this treatment, the surfaces of the PHF and PPeF samples were fairly comparable to the ones observed in the as-casted cases (Figure $1 \mathbf{d}$ ) and e)), also showing similar $R_{\mathrm{a}}$ values (Table 3). However, the fast-cooled PBF surface topography changed considerably, as evidenced by the disappearance of the circular inclusions (Figure $1 \mathbf{f}$ )). In fact, the thin film now exhibits a continuous surface with a roughness of $\sim 1 \mathrm{~nm}$. A critical look into Figure 1 f) allowed observing that there were some small nanometric features on the fast-cooled PBF thin film, comparable to those observed in the PHF samples. Then, we conclude that the peculiar nanostructured surface of the as-casted PBF was related to the presence of metastable features that disappeared after heating. Also, we highlight the fast-cooling process performed on the PHF and PBF thin films did not result in fully amorphous samples (see $R_{\mathrm{a}}$ values), but in the presence of aggregates possibly related to small crystalline features. This idea is in line with 
the previously reported bulk thermal characterization, where fast-cooled PHF and PBF samples presented non-negligible crystallinity values $[11,39]$.

The right column in Figure 1 shows the AFM height images for slow-cooled thin films. This thermal treatment resulted in differences in the surface topography of the thin films, based on the glycolic subunit length of the polymers. Slow-cooled PHF and PBF thin films, showed the development of clearly defined polymer crystals (Figure 1 g) and i)). In particular, PHF developed large structures, typically observed for polymer spherulites $[36,58]$, while PBF showed smaller needle-like crystals. The formation of these nanostructures was also accompanied by an important change in the surfaces' roughness. For these two polymers, we quantified a doubling of $R_{\mathrm{a}}$, comparing to the previously discussed treatments (Table 3). In the case of the slow-cooled PPeF, the resulting AFM image was continuous with a low roughness, as for the other PPeF thin films so far discussed. In other words, the PPeF thin film did not crystallize even during slow cooling from the melt. This fact is expected considering previous reports on this polymer $[9,11]$. Nonetheless, we highlight that even after annealing at high temperatures $\left(180^{\circ} \mathrm{C}=T_{\mathrm{g}}^{\mathrm{PPeF}}+165^{\circ} \mathrm{C}\right)$, the PPeF sample did not undergo dewetting. Then, the PPeF thin film presented a high mechanical stability even though in the rubbery state and in a confined geometry. This finding is in line with the interesting bulk properties of furanbased polymers, particularly those observed for PPeF in recent works [9, 11, 40]. In fact, Martínez-Tong and collaborators showed that this polymer does not reach Newtonian flow at high temperatures $\left(120-150^{\circ} \mathrm{C}\right)$, as observed by rheology measurements [40]. Other authors have proposed the existence of interchain interactions in different furan-based polymers [11, 59]. In the particular case of PPeF, Guidotti and collaborators linked the formation of a mesophase to interchain interactions [9]. All these reports in recent literature support the fact that PPeF thin films can withstand high temperature treatments without dewetting. 
Furthermore, we evaluated the possible nanostructure formation/evolution on the poly(alkylene 2,5-furanoate) thin films, after aging at room temperature. The as-casted samples were stored in a desiccator and evaluated progressively. The PHF and PBF surfaces presented identical surface topographies as the ones observed for the as-casted cases, even after aging for 6 months (Figure S2). This result is somehow expected for PBF, due to $T_{\mathrm{g}}^{\mathrm{PBF}}>\mathrm{RT}$, and thus the polymer chains have not enough mobility to reorganize at room temperature. In the case of PHF, the thermal transitions indicate that polymer chains could rearrange at room temperature $\left(T_{\mathrm{g}}^{\mathrm{PHF}}<\mathrm{RT}\right)$. Consequently, the lack of evolution of surface topography after this long time, is quite interesting. This result can be explained as due to the presence of crystalline domains that exert restrictions on the neighboring amorphous chains. The reduction in their mobility prevents further large scale rearrangement of the remaining amorphous regions, even if in the rubbery state. In this way, the aging experiment further supports the semicrystalline nature of the as-casted PHF thin film, despite its surface does not show the "usual" spherulitic geometry, this last instead, developed by slow cooling from the melt (Figure $1 \mathbf{g})$ ). 

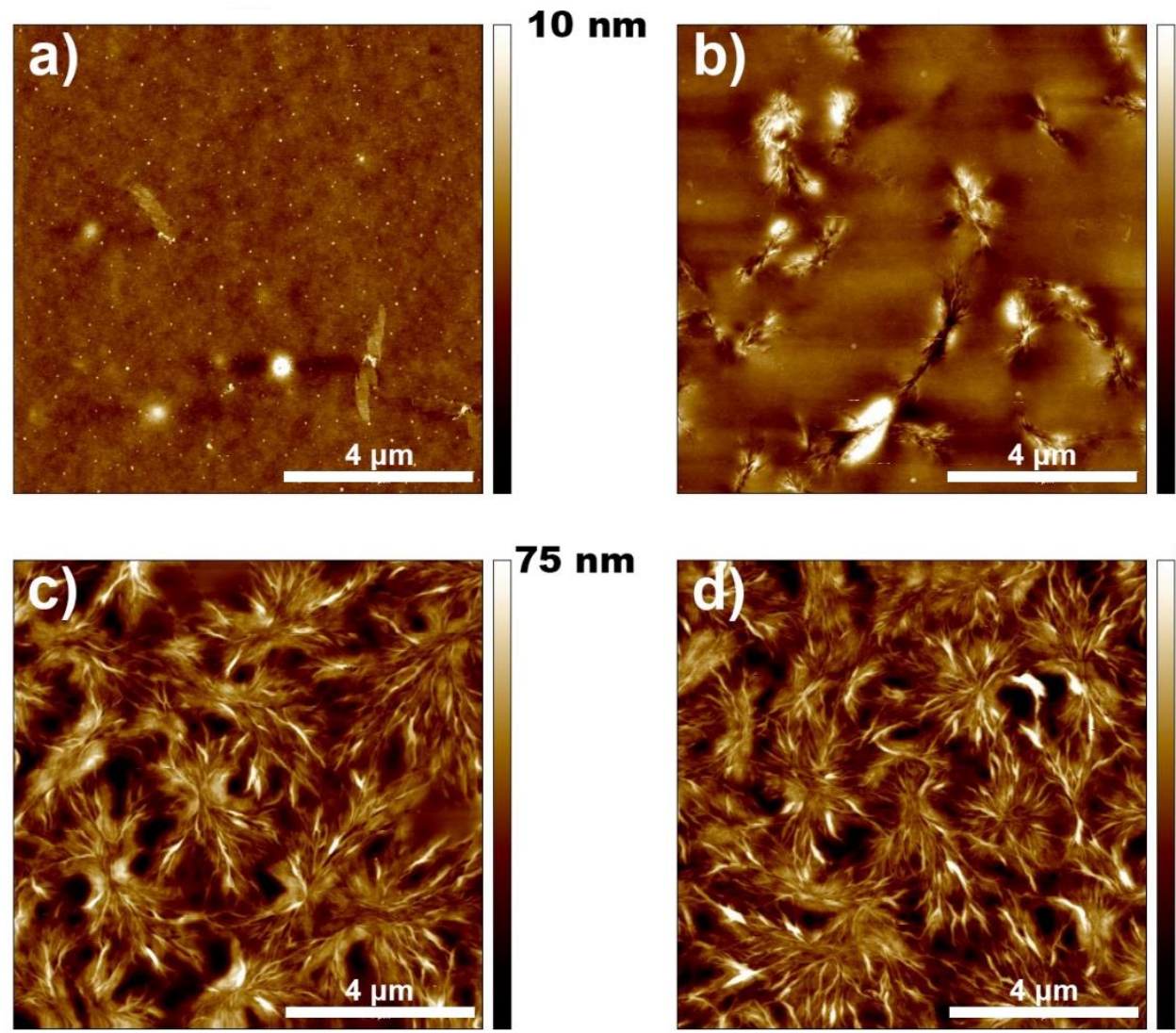

\section{$75 \mathrm{~nm}$}

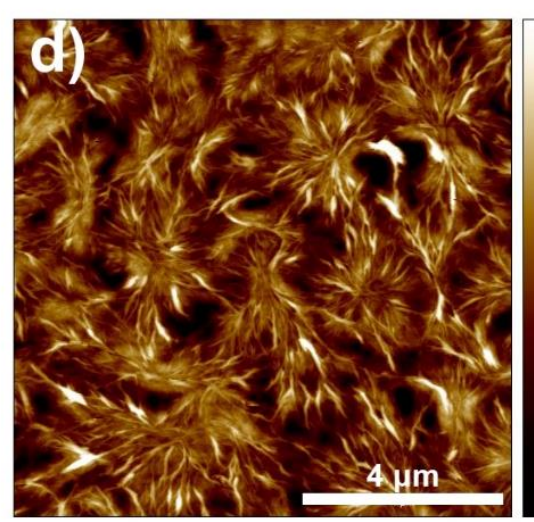

$75 \mathrm{~nm}$

Figure 2. $10 \times 10$ micrometers AFM height images of the poly(pentamethylene 2,5-furanoate) thin films showing the evolution of the crystallization throughout 1 year: a) as-casted, b) 3 months, c) 6 months and d) 1 year aged films.

Figure 2 shows the surface evolution of the as-casted PPeF thin film, progressively evaluated during aging throughout 1 year. In this case, we observed the growth of polymer nanostructures randomly distributed all over the surface, resembling polymer crystals. The nanostructure formation was accompanied by a strong increase in $R_{\mathrm{a}}$, over a factor 10 (Table 3). From Figure 2, one can see that the crystallization kinetics was very slow and the first small crystal nuclei were visible only after three months of annealing at room temperature. Besides, the structural development seems to be completed nearly after 6 months, presenting the 6 months- and 1 year-aged films practically the same features. The slow crystallization kinetics in the PPeF thin films are in line with bulk calorimetric studies on this polymer [40]. In fact, in the present case 
we observed nanostructures resembling those of the non-symmetrical spherulites typically developed by polymers at low undercooling degree. This is the situation for the PPeF nanostructures, where the crystallization temperature $\left(25^{\circ} \mathrm{C}\right)$ was relatively close to the bulk melting temperature $\left(42^{\circ} \mathrm{C}\right.$, Table 1$)$.

\subsection{Monitoring PBF crystallization: effect of film thickness and thermal history.}

As it can be inferred from the calorimetric studies [39] and our present AFM results, PBF showed a more rich and complex thermal behavior, with a crystallinity strongly dependent on the thermal history. Therefore, in order to study influence of the thickness on the nanostructure growth, we prepared two extra PBF thin films of different thickness (54 nm and $14 \mathrm{~nm}$, plus the already discussed $112 \mathrm{~nm}$ one). The different thicknesses were achieved from the original PBF solution by adding appropriate volumes of chloroform, i.e., preparing more dilute solutions. These samples were subjected to three thermal protocols. The first one was the already discussed fast-cooling protocol. In the second treatment, the PBF samples were subjected to non-isothermal melt-crystallization by cooling the thin films from $180{ }^{\circ} \mathrm{C}$ to room temperature at a moderate rate of $5 \mathrm{~K} \cdot \mathrm{min}^{-1}$. In case of the third treatment, the PBF samples were cooled down from $180{ }^{\circ} \mathrm{C}$ to $155{ }^{\circ} \mathrm{C}$, at a controlled $3 \mathrm{~K} \cdot \mathrm{min}^{-1}$ rate. We held this temperature for $1 \mathrm{~h}$, in order to promote isothermal crystallization, and then we cooled down the samples to room temperature, at $3 \mathrm{~K} \cdot \mathrm{min}^{-1}$. Figure 3 presents the AFM height images showing the topological features developed on each PBF thin film, after the corresponding thermal treatment. 


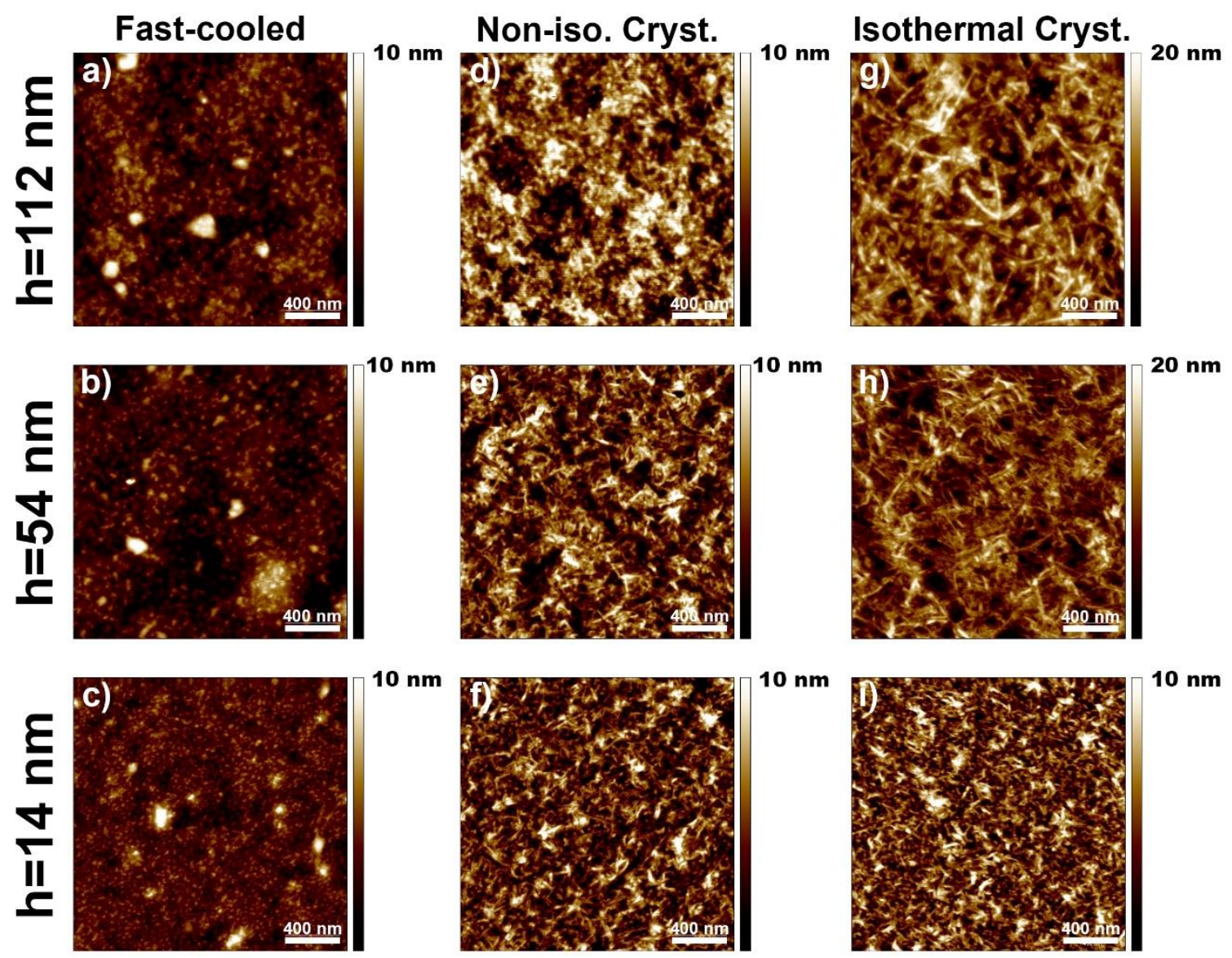

Figure 3. AFM height images of the PBF thin films of several thickness subjected to different thermal protocols: a)-c) fast-cooled films, d)-f) non-isothermally crystallized samples, and h)i) isothermally crystallized films.

Table 4. Mean surface roughness values of the PBF thin films with different thickness subjected to the above mentioned thermal treatments.

\begin{tabular}{l|ccc} 
& \multicolumn{3}{c}{$R_{\mathrm{a}}(\mathrm{nm})$} \\
\cline { 2 - 4 } Sample & Fast-cooled & Non-Iso. Cryst. & Isothermal Cryst. \\
\hline$h=112 \mathrm{~nm}$ & 1.03 & 1.80 & 2.70 \\
$h=54 \mathrm{~nm}$ & 1.00 & 1.65 & 2.52 \\
$h=14 \mathrm{~nm}$ & 0.82 & 1.56 & 1.56
\end{tabular}

In general, we observed that all PBF thin films, even the thinnest ones, were able to withstand the thermal treatments and showed no dewetting. For the fast-cooled films (Figure 3 a)-c)) we 
observed relatively flat surfaces, showing small aggregates similar to the ones previously detected and discussed for the $112 \mathrm{~nm}$ PBF film. The quantified $R_{\mathrm{a}}$ values were almost constant for the $112 \mathrm{~nm}$ and $54 \mathrm{~nm}$ films, showing only a small decrease for the thinnest sample (Table 4). The AFM height images of the isothermally crystallized thin films (Figure $3 \mathbf{g}$ )-i)) also presented crystalline nanostructures in all the cases. Again, the features were needle-like for all thicknesses, even though some interesting differences can be observed. In the particular case of the thicker films $(h=112 \mathrm{~nm}$ and $54 \mathrm{~nm}$ ), this protocol evidenced the presence of nanostructures about $2 x$ longer than those found for the slow-cool treatment (see Figure 1 d)e) vs. g)-h)). This indicated that the isothermal treatment promoted the crystalline growth, as expected. These differences are also reflected in the remarkable change of $R_{\mathrm{a}}$ values for the thicker films, when comparing the non-isothermal vs. isothermally crystallized samples. Interestingly, for the $14 \mathrm{~nm}$ films, there was not a perceptible change in the size of the crystals as a function of thermal history, with the mean surface roughness remaining almost unaltered

(Table 4). The observed distinct behavior of thinnest PBF thin film can be understood taking into consideration the hindrances imposed by confinement effects in this ultra-thin case, as extensively reported in the literature [29, 32].

\subsection{Nanomechanical properties of poly(alkylene 2,5 -furanoate) thin films.}

Now, we focus our attention on the effect of thermal history and nanostructure development on the nanomechanical properties of poly(alkylene 2,5-furanoate) thin films. For this study, we selected the thickest films $(h>100 \mathrm{~nm}$ ), to avoid the possible influence of the supporting substrate on the measurements. The obtained results are presented in Figure 4 and 5, where all nanomechanical maps are related exclusively to the mechanical contrast. In this so-called "semiquantitative" mapping, we are confident on the contrast scales shown, i.e. the variation of the property within a specific range, but not on the absolute mean value of the maps. 
Figure 4 shows the surface topography and dynamic mechanical maps (PF-QNM) for fastcooled poly(alkylene 2,5-furanoate) thin films. As discussed in the lines above, these fastcooled samples resulted in thin films with the lower nanostructure development on their surfaces. In these cases, PHF and PBF have a certain degree of crystallinity, while PPeF is in an amorphous state (see Section 3.1 for further details). We observed that all the fast-cooled thin films presented homogenous nanomechanical maps. This is an indication that there are not zones with preferential mechanical properties on the samples' surfaces. Moreover, the polymer crystals, if present, have to be quite small and uniformly distributed throughout the polymer surface, so that their presence does not result in nanomechanical contrast. In detail, the fastcooled PHF and PBF thin films showed stiffness variations of about $4 \mathrm{~N} \cdot \mathrm{m}^{-1}$ through the film surface, while PPeF presented an almost constant stiffness, with variations below $2 \mathrm{~N} \cdot \mathrm{m}^{-1}$. For all the fast-cooled thin films, the adhesion force maps were similar and mostly constant within each sample, showing a variance of $1 \mathrm{nN}$. Please, beware that in the case of the fast-cooled PPeF, the material's response should be viscoelastic since we performed the measurements at $T>T_{\text {g-PPeF }}$. 

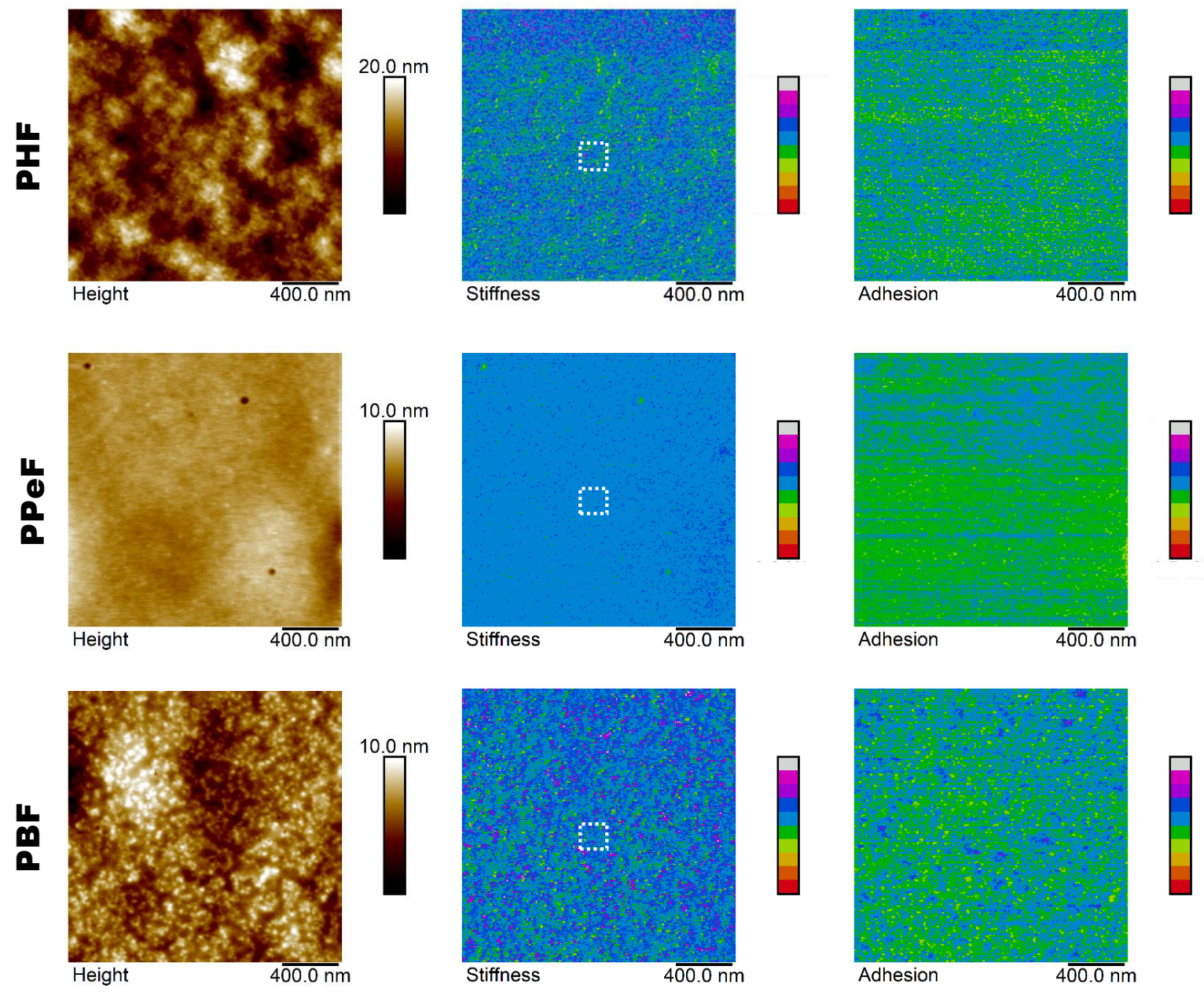

Figure 4. PF-QNM maps of fast-cooled PHF, PPeF, and PBF thin films. For the stiffness maps, each color in the colorbar represents a $2 \mathrm{~N} \cdot \mathrm{m}^{-1}$ variation, while for the adhesion force maps, it represents a $1 \mathrm{nN}$ variation. The white squares in the stiffness maps indicate the zones where force spectroscopy analysis was performed, as detailed in the main text.

Figure 5 shows the PF-QNM maps for the most crystalline poly(alkylene 2,5-furanoate) thin films. In this case, we selected different thermal treatments depending on the samples' nature, in order to allow having all the thin films with the largest development of well-defined polymer crystals on the surface. Please beware that in the following lines, we will refer to this set of samples as "semicrystalline" for the sake of simplicity. 
In detail, semicrystalline PHF and PBF samples were selected as those prepared by slowcooling from the melt $\left(180^{\circ} \mathrm{C}\right.$ to $\mathrm{RT}$, at $\left.3 \mathrm{~K} \cdot \mathrm{min}^{-1}\right)$, while for the PPeF film we studied the sample aged for 1 year at room conditions. As observed in Figure 5, most of these PF-QNM maps presented a well-defined contrast, indicating mechanical differences throughout the surfaces. For all the samples, the stiffness maps showed variations up to $18 \mathrm{~N} \cdot \mathrm{m}^{-1}$, readily attributable to the further development of the polymers' crystalline phase. However, there were different responses concerning the adhesion force. In this case, the PHF and PPeF semicrystalline thin films showed variations of up to $6 \mathrm{nN}$, while PBF showed variations of just $2 \mathrm{nN}$, analogously to the fast-cooled case. This result could be explained on the basis of the different nature of the amorphous fractions at room temperature, rubbery for PHF and PPeF, and glassy for PBF. In this view, a higher dispersion of the adhesion force values can be expected in the former polymers, since their film surface alternate stiff crystal regions with softer rubbery ones. On the contrary, lower variations of adhesion force for PBF are in line with the coexistence of two rigid domains, the crystals and the glassy amorphous phase. 

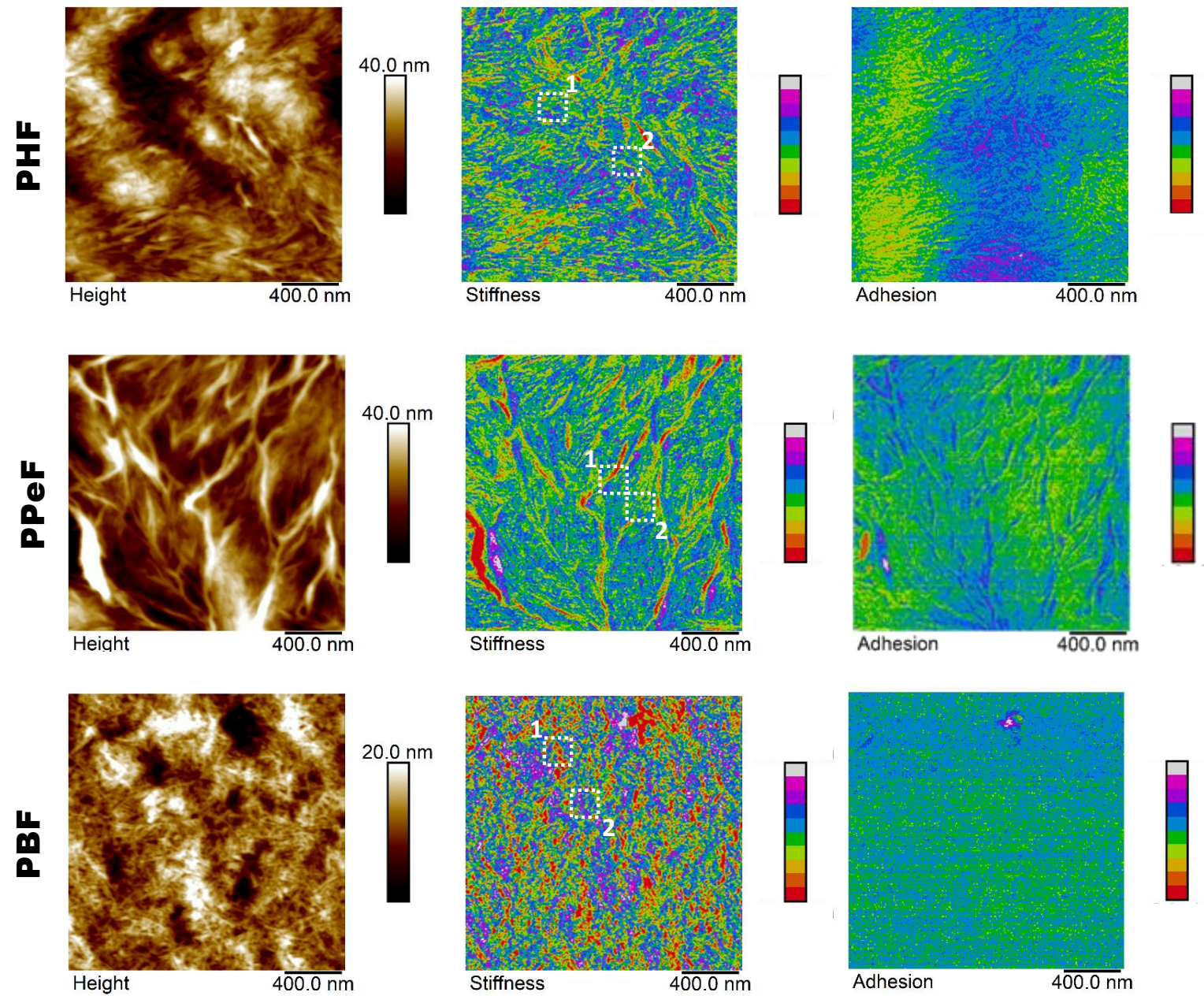

Figure 5. PF-QNM maps of semicrystalline PHF, PPeF, and PBF thin films. For the stiffness maps, each color in the colorbar represents a $2 \mathrm{~N} \cdot \mathrm{m}^{-1}$ variation, while for the adhesion force maps, it represents a $1 \mathrm{nN}$ variation. The white squares in the stiffness maps indicate the different zones where force spectroscopy analysis was performed, as detailed in the main text.

To provide an in-depth nanomechanical study, we performed force spectroscopy measurements on selected regions of the fast-cooled and semicrystalline thin films just discussed. Although we explored several points on the films' surfaces, in Figure 6 we present representative force spectroscopy curves, taken at the zones indicated in Figure 4 and Figure 5 by the different squares in the stiffness maps. In the particular case of the more crystalline poly(alkylene 2,5-furanoate) thin films, we present force spectroscopy measurements on two different zones (highlighted by the numbers $1 \& 2$ in Figure 5). Those zones were selected by 
considering the observed PF-QNM contrast. The quantitative values obtained from the force spectroscopy analysis are summarized in Table 5.

Fast-cooled
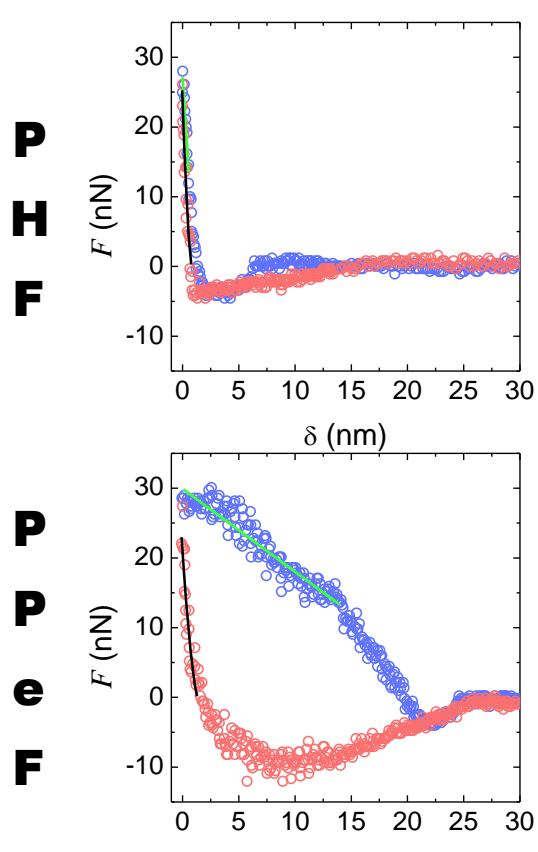

$\delta(\mathrm{nm})$

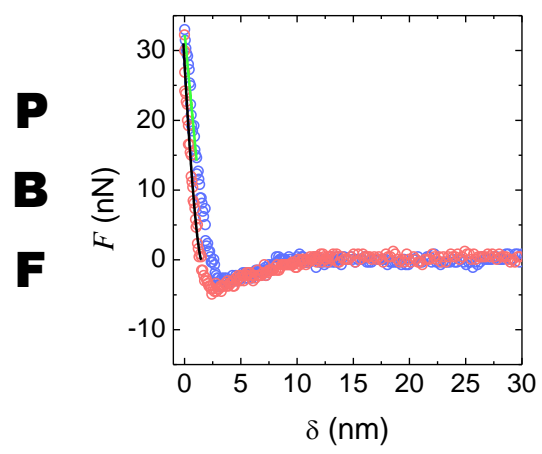

Semicryst

Zone 1

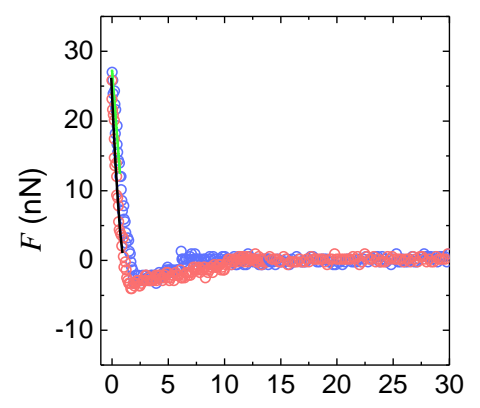

$\delta(\mathrm{nm})$

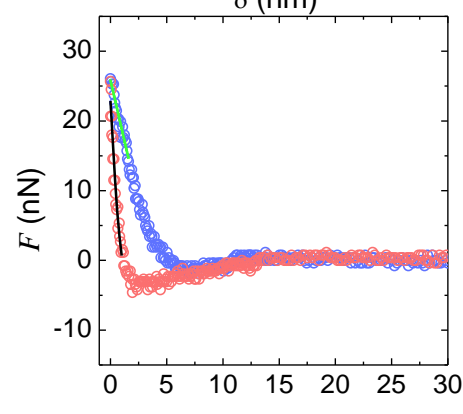

$\delta(\mathrm{nm})$

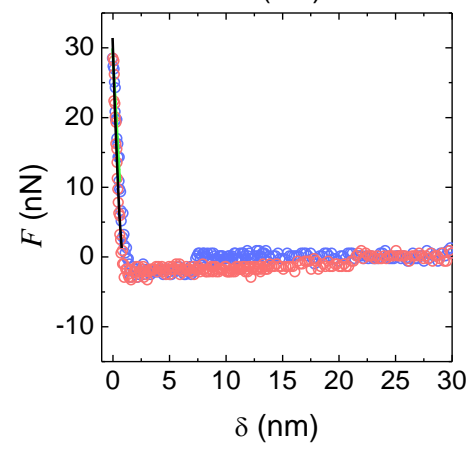

Semicryst

Zone 2

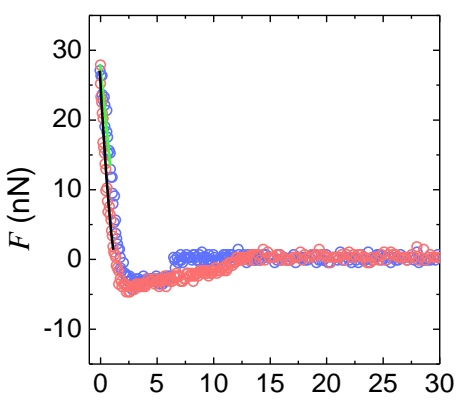

$\delta(\mathrm{nm})$

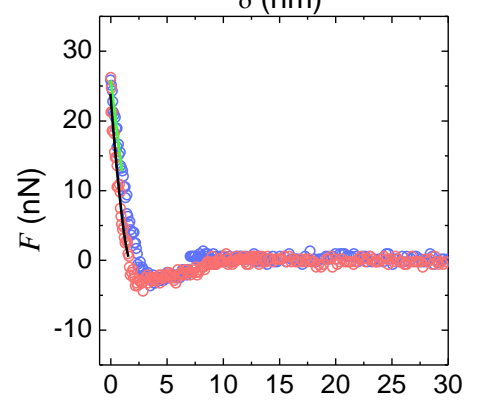

$\delta(\mathrm{nm})$

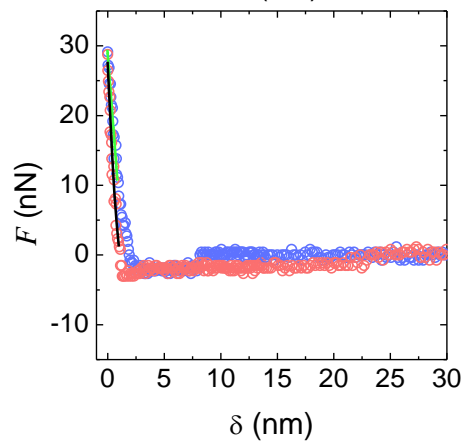

Figure 6. Force spectroscopy results for the different poly(alkylene 2,5-furanoate) thin films. Blue dots correspond to the tip-sample approach curve, while orange dots to the tip-sample retract curve (see Supporting Information for further details). The continuous lines in these plots correspond to the fittings explained in the Experimental Section. Bright green lines correspond to the stiffness fitting, and black lines to the Hertz model for calculation of $E_{\mathrm{Y}}$. The so-called Zones $1 \& 2$, in the semicrystalline cases, represent the areas where the force spectroscopy analysis was performed, indicated in Figure 5 by the white numbered squares. For simplicity, in the force spectroscopy curves, we show only 1 every 5 points in the interacting zones of the curves, and 1 every 10 points in the non-interacting areas. Fittings were carried out using the whole dataset. 
We begin our analysis with the PHF and PBF thin films, i.e., those able to crystallize readily. These fast-cooled samples presented stiffness values of the same order of magnitude, being 18 $\mathrm{N} \cdot \mathrm{m}^{-1}$ for PHF, and $22 \mathrm{~N} \cdot \mathrm{m}^{-1}$ for PBF. The slightly higher stiffness observed for PBF was also accompanied by a lower indentation depth (1.4 nm vs $2.4 \mathrm{~nm}$, respectively). These results indicated that, during tip-sample approach, the PBF surface imposed higher resistance to penetration, compared to that of the PHF. From the tip-sample retract curve, we found the same adhesion force value of $4.5 \mathrm{nN}$ for both samples. Finally, the Young's modulus $\left(E_{\mathrm{Y}}\right)$ of fastcooled PHF was 1.1 GPa, while for PBF we obtained a value of 2.4 GPa. Overall speaking, our force spectroscopy study indicated that the PBF fast-cooled thin film presented enhanced mechanical properties respect to the PHF one, being characterized by higher modulus and stiffness.

The semicrystalline PHF and PBF thin films presented different stiffness values depending on the probed zones, a result in line with the contrast observed in the PF-QNM maps (Figure 5). As for the fast-cooled case, the PBF sample presented higher mean stiffness than the PHF, nonetheless, the indentation depths are now quite comparable and around $1.5 \mathrm{~nm}$. Also, both semicrystalline samples presented a decrease in the adhesion force values, when compared to the fast-cooled samples, being this fact more pronounced for the PBF thin film. Finally, the semicrystalline PHF thin film presented the same Young's modulus value regardless of the explored area, being both values higher than the one calculated for the fast-cooled case. In the case of the semicrystalline PBF thin film, we observed different Young's modulus values according to the explored areas. However, contrary to PHF, there is not a clear trend about the effect of the crystalline nanostructures on $E_{\mathrm{Y}}$. 
The so-far presented results can be related to the different thermal and structural properties of these polymers. At room temperature, both thin films are expected to have some degree of crystallinity for both thermal histories, as previously discussed in this manuscript and as inferred from the reported bulk DSC results. However, the glass transition temperature of PHF is below room temperature, while the one for PBF is above it. Then, during force spectroscopy measurements, the amorphous phase of PHF is rubbery and softer than the one of PBF, which is glassy. This is line with the differences observed between these two materials in both stiffness and indentation depth. The development of well-defined crystalline nanostructures, by slow-cooling from the melt, resulted in enhanced mechanical properties for both thin films. This can be related to a lower fraction of amorphous phase, replaced by polymer crystals. We highlight that PHF developed crystalline nanostructures resembling polymer spherulites, while PBF showed needle-like structures. These geometrical differences are expected to have an impact on the nanomechanical properties. In fact, the semicrystalline PBF thin film showed a lower adhesion force than $\mathrm{PHF}$, while at the same time presented a more pronounced $E_{\mathrm{Y}}$ contrast among probed areas. It is also worth noting that the Young's modulus values found for these two polymers are slightly higher (about the double) than the ones reported in the literature for bulk films, determined by means of tensile tests $[8,10,38]$. Variations in the Young's modulus, between bulk and nanometric tests have been previously reported in the literature, and related mostly to the different measurement protocols [47, 48]. Nonetheless, we highlight that our reported moduli are in the same order of magnitude as those reported previously $[8,10,38]$, and that the ratio between PBF and PHF $E_{\mathrm{Y}}$ values remained the same $(\sim 1.8 \times)$, regardless of whether bulk or nanomechanical tests are being considered. 
Table 5. Force spectroscopy results. For semicrystalline samples, Zones $1 \& 2$ are those indicated in Figure 5 by the different numbered squares.

\begin{tabular}{l|cccc}
\cline { 2 - 5 } $\begin{array}{l}\text { Sample } \\
\text { treatment }\end{array}$ & $\begin{array}{c}\text { Stiffness } \\
\left(\mathrm{N} \cdot \mathrm{m}^{-1}\right)\end{array}$ & $\begin{array}{c}\delta_{\mathrm{i}} \\
(\mathrm{nm})\end{array}$ & $\begin{array}{c}F_{\mathrm{adh}} \\
(\mathrm{nN})\end{array}$ & $\begin{array}{c}E_{Y} \\
(\mathrm{GPa})\end{array}$ \\
\hline $\begin{array}{l}P H F \\
\text { fast-cooled }\end{array}$ & $18 \pm 1$ & $2.4 \pm 0.2$ & $4.5 \pm 0.2$ & $1.1 \pm 0.2$ \\
$\begin{array}{l}P H F \\
\text { Semicryst-Zone 1 }\end{array}$ & $22 \pm 1$ & $1.5 \pm 0.2$ & $3.3 \pm 0.2$ & $1.6 \pm 0.2$ \\
$\begin{array}{l}P H F \\
\text { Semicryst-Zone 2 }\end{array}$ & $16 \pm 1$ & $1.7 \pm 0.2$ & $3.9 \pm 0.2$ & $1.3 \pm 0.2$ \\
\hline $\begin{array}{l}\text { PPeF } \\
\text { fast-cooled }\end{array}$ & $1.2 \pm 0.3$ & $20 \pm 1$ & $10 \pm 0.5$ & $0.5 \pm 0.2$ \\
$\begin{array}{l}\text { PPeF } \\
\text { Semicryst-Zone 1 }\end{array}$ & $6 \pm 1$ & $5 \pm 0.5$ & $3.1 \pm 0.2$ & $1.8 \pm 0.2$ \\
$\begin{array}{l}\text { PPeF } \\
\text { Semicryst-Zone 2 }\end{array}$ & $13 \pm 1$ & $2.4 \pm 0.3$ & $3.3 \pm 0.2$ & $0.8 \pm 0.2$ \\
\hline $\begin{array}{l}\text { PBF } \\
\text { fast-cooled }\end{array}$ & $22 \pm 1$ & $1.4 \pm 0.2$ & $4.5 \pm 0.2$ & $2.4 \pm 0.2$ \\
$\begin{array}{l}P B F \\
\text { Semicryst-Zone 1 }\end{array}$ & $29 \pm 1$ & $1.3 \pm 0.2$ & $1.6 \pm 0.2$ & $2.7 \pm 0.2$ \\
$\begin{array}{l}\text { PBF } \\
\text { Semicryst-Zone 2 }\end{array}$ & $22 \pm 1$ & $2.0 \pm 0.2$ & $2.0 \pm 0.2$ & $1.9 \pm 0.2$ \\
\end{tabular}

The force spectroscopy measurements on the PPeF thin films, both fast-cooled and semicrystalline cases, gave different results compared to the samples discussed so far. The fastcooled force-distance curve of PPeF showed a quite complex behavior, as it is immediately visible from Figure 6. During approach (blue points), we found a surface stiffness of $\sim 1 \mathrm{~N} \cdot \mathrm{m}^{-}$ ${ }^{1}$, with a deep indentation of $\sim 20 \mathrm{~nm}$. These values are about 1 order of magnitude different from the ones found for PHF and PBF. The semicrystalline PPeF thin film showed an increase in the stiffness, to values above $6 \mathrm{~N} \cdot \mathrm{m}^{-1}$, with a concomitant decrease in the indentation depth. We found differences in these parameters depending on the probed zones, in line with the PFQNM maps (Figure 5). However, the quantitative values of stiffness and $\delta_{\mathrm{i}}$ indicated that the sample surface resistance was lower, about halved, than those evaluated for semicrystalline 
PHF and PBF. This result might be related to the crystallinity of these polymers. Using DSC data, the crystallinity $\left(\chi_{\mathrm{c}}\right)$ can be calculated as $\chi_{\mathrm{c}}=\Delta H_{\mathrm{m}} / \Delta H_{\mathrm{m}}^{0}$, where $\Delta H_{\mathrm{m}}$ is the melting heat of the semicrystalline samples, summarized in Table 1, while $\Delta H_{\mathrm{m}}^{0}$ the melting heat of the fully crystalline polymers. Using this approach, we calculated a $\chi_{\mathrm{c}}=0.28\left(\Delta H_{\mathrm{m}}^{0}=143 \mathrm{~J} / \mathrm{g}\right.$, [60]) for PHF, and $\chi_{\mathrm{c}}=0.29\left(\Delta H_{\mathrm{m}}^{0}=129 \mathrm{~J} / \mathrm{g},[61]\right)$ for PBF. In the case PPeF, there are not any reports on its value of $\Delta H_{\mathrm{m}}^{0}$, complicating the calculation of $\chi_{\mathrm{c}}$ by DSC, as thoroughly discussed in Ref[40]. However, considering its lower $\Delta H_{\mathrm{m}}$ value (Table 1) in combination with the AFM images (Figure 2), PPeF presents a low crystallizing ability, which might turn into a lesser and less perfect crystalline phase, i.e., much lower $\chi_{\mathrm{c}}$ and $T_{\mathrm{m}}$ as compared to PHF and PBF [40]. Then, in this scenario, the low crystallinity PPeF surface would oppose less resistance, allowing deeper penetration of the AFM tip during approach, as observed in the force spectroscopy results.

The fast-cooled PPeF force-distance curve showed an important hysteresis, as observed by the lack of overlapping between approach and retract points during indentation. The area under this curve is defined as "plasticity index" of the sample, and it is related to the energy dissipation of the material during the load/unload cycles (i.e., tip approach and retraction) [62]. Then, comparing with the other two poly(alkylene 2,5-furanote)s under study, PPeF shows an important energy dissipation at room temperature. All these results can be somehow expected considering that $T_{\mathrm{g}}$ of $\mathrm{PPeF}$ is below room temperature, and that the polymer has very slow crystallization kinetics, i.e., the fast-cooled sample is rubbery and viscoelastic, while being probed. It worth of remark that the approach curve the fast-cooled PPeF (Figure 6) presents a peculiar shape with a slope that is not monotonously increasing, contrary to usual observations [63]. This particular finding might be an indication of the suggested presence of hydrogen bonds in PPeF, as previously discussed [9]. 
The hysteresis of the force-distance curve was drastically reduced, for the semicrystalline thin films, compared to the fast-cooled one. There was still some energy dissipation, but significantly lower than that observed for the fast-cooled sample. We highlight that PPeF was the only poly(alkylene 2,5-furanote) sample where an energy dissipation signature was present. This is an interesting result, specially comparing with the results obtained for the semicrystalline PHF thin film. As for PPeF, PHF has a $T_{\mathrm{g}}$ below room temperature, however, in this case, no load/unload hysteresis was recorded. Since energy dissipation should arise mostly from the amorphous fraction in the semicrystalline polymers, our nanomechanical studies suggest that amorphous fraction packing in these two situations would be different. Once again, this result could correspond to the presence of less perfect crystals in the PPeF thin film, compared to the PHF. However, we highlight that the topography images did not show shocking differences in the crystalline morphology between these two polymers. This might indicate that our present force spectroscopy measurements are influenced not only by the surface properties, but that there also contributions arising from the sample's volume.

During tip-sample retract (orange symbols), we quantified an adhesion force of $10 \mathrm{nN}$ for fast-cooled PPeF thin films. This was the highest adhesion force value found, representing an 2x increase if compared to PHF and PBF. Using the retraction curves, we also calculated the Young's modulus for the PPeF thin films. The fast-cooled PPeF sample resulted in a $E_{\mathrm{Y}}$ value of $0.5 \mathrm{GPa}$. These nanomechanical properties of PPeF changed significantly after nanostructure development also. The semicrystalline PPeF thin film presented a decreased in the adhesion force. Now, the obtained values are similar to those found for the slow-cooled $\mathrm{PHF}$, around $3 \mathrm{nN}$. The calculated $E_{\mathrm{Y}}$ showed an increase after nanostructure formation, with values depending on the probed area. Then, nanostructure formation on PPeF by polymer crystallization after aging, led to enhanced mechanical properties, closer to the ones found for 
PHF and PBF. Comparing to previous results, we cannot fail to highlight the obtained Young's modulus for fast-cooled PPeF is about 50 times higher than the one quantified by tensile test measurements in the bulk [9]. However, the nanomechanical modulus value is close to the one that could be expected for a mechanical alpha relaxation showing maximum losses (half of the step) close to room temperature. We can support this idea by looking at recently reported dielectric relaxation studies on fast cooled PPeF. In this case, at $25{ }^{\circ} \mathrm{C}$, the dielectric alpha relaxation showed its characteristic frequency between $1 \mathrm{~Hz}-100 \mathrm{~Hz}$ [40], a process that it linked to the mechanical relaxation of the material.

The nanomechanical properties of the presented furan-based thin films are fairly comparable to those found for bulk PPF, using an AFM-based force spectroscopy method [26]. This demonstrates that poly(alkylene 2,5-furanoate) prepared in thin film geometry preserve their surface mechanical properties, despite the differences in the experimental procedure. Finally, we also stand out that the mechanical properties of the poly(alkylene 2,5-furanoate) thin films are comparable to those found for terephthalic-based polymers, using nanomechanical methods. Recently, Rodríguez-Beltrán and collaborators reported the local mechanical properties of poly(ethylene terephthalate) (PET) from PF-QNM maps [47]. In their work, the authors reported a value of $E_{\mathrm{Y}}$ of $1.6 \mathrm{GPa}$, an adhesion force of $\sim 7 \mathrm{nN}$, and a sample deformation close to $4 \mathrm{~nm}$. Comparing with our present results, we notice that poly(alkylene 2,5-furanoate) thin films under investigation showed Young's moduli values on the same order of magnitude, but lower adhesion force and lower deformation, as expected if we consider that the polymers under study contain more flexible glycol subunits. This comparison further proves that the furan-based polymers presented in the present paper can be valuable alternatives to PET. 


\section{Conclusions}

In overall, our results represent an initial step into the possible use of poly(alkylene 2,5furanoate)s in confined and nanoscale geometries, as thin films. For all the studied polymers, we were able to obtain continuous films, with thicknesses below $150 \mathrm{~nm}$, showing no signs of dewetting. In the specific case of PBF, we were able to decrease film thickness down to $14 \mathrm{~nm}$, without compromising the films' integrity. The growth of polymer crystals was studied for all films as a function of different thermal treatments. Samples containing a C-even-numbered glycolic subunit (PHF and PBF) showed the formation of different crystalline nanostructures depending on the thermal treatment. PPeF, being the only polymer with a C-odd-numbered glycolic subunit, showed no formation of crystals in regular laboratory times. However, after aging for several months, we were able to observe the growth of crystalline nanostructures on the PPeF surface too. The nanomechanical properties of the poly(alkylene 2,5-furanoate) thin films were studied combining PF-QNM and force spectroscopy measurements. We determined that semicrystalline samples presented nanomechanical contrast, as evidenced by PF-QNM maps. The quantitative mechanical properties were extracted from the force spectroscopy analysis. In general, the studied samples had Young's moduli values on the order of the GPa, adhesion forces around 2-5 $\mathrm{nN}$, and indentation depths around $1.5 \mathrm{~nm}$, with the exception of the amorphous PPeF thin film, where a complex behavior was observed and discussed. The nanomechanical properties of our materials were shown to be comparable to those found for macroscopic furan-based polymers, and for PET. This pointed out that the preparation of poly(alkylene 2,5-furanoate) thin films preserve the good mechanical properties of bulk materials and are comparable to those shown by petrochemical polymers such as PET.

\section{AUTHOR INFORMATION}

\section{Corresponding Authors}


*E-mail:m.soccio@unibo.it. Phone:+390512090360.

*E-mail: danielenrique.martinezt@ehu.eus. Phone:+34 943018824.

\section{ACKNOWLEDGMENTS}

D. E. M acknowledges financial support from "Ministerio de Ciencia, Innovación y Universidades" (MCIU), via a “Juan de la Cierva - Incorporación” grant (IJCI-2017-31600). B. R. and A. A. acknowledge funding from Basque Government (IT-1175-19). G.G., M.S. and N.L. acknowledge the Italian Ministry of University and Research. This publication is part of the framework COST Action FUR4Sustain, CA18220, supported by COST (European Cooperation in Science and Technology).

\section{REFERENCES}

[1] S.K. Burgess, J.E. Leisen, B.E. Kraftschik, C.R. Mubarak, R.M. Kriegel, W.J. Koros, Chain Mobility, Thermal, and Mechanical Properties of Poly(ethylene furanoate) Compared to Poly(ethylene terephthalate), Macromolecules 47(4) (2014) 1383-1391.

[2] G.Z. Papageorgiou, V. Tsanaktsis, D.N. Bikiaris, Synthesis of poly(ethylene furandicarboxylate) polyester using monomers derived from renewable resources: thermal behavior comparison with PET and PEN, Physical Chemistry Chemical Physics 16(17) (2014) 7946-7958.

[3] L. Papadopoulos, A. Magaziotis, M. Nerantzaki, Z. Terzopoulou, G.Z. Papageorgiou, D.N. Bikiaris, Synthesis and characterization of novel poly(ethylene furanoate-co-adipate) random copolyesters with enhanced biodegradability, Polymer Degradation and Stability 156 (2018) $32-42$.

[4] J.-G. Rosenboom, D.K. Hohl, P. Fleckenstein, G. Storti, M. Morbidelli, Bottle-grade polyethylene furanoate from ring-opening polymerisation of cyclic oligomers, Nature Communications 9(1) (2018) 2701.

[5] G.Z. Papageorgiou, D.G. Papageorgiou, V. Tsanaktsis, D.N. Bikiaris, Synthesis of the biobased polyester poly(propylene 2,5-furan dicarboxylate). Comparison of thermal behavior and solid state structure with its terephthalate and naphthalate homologues, Polymer 62 (2015) 2838.

[6] G. Guidotti, M. Soccio, N. Lotti, M. Gazzano, V. Siracusa, A. Munari, Poly(propylene 2,5thiophenedicarboxylate) vs. Poly(propylene 2,5-furandicarboxylate): Two Examples of High Gas Barrier Bio-Based Polyesters, Polymers 10(7) (2018) 785.

[7] G. Guidotti, L. Genovese, M. Soccio, M. Gigli, A. Munari, V. Siracusa, N. Lotti, Block Copolyesters Containing 2,5-Furan and trans-1,4-Cyclohexane Subunits with Outstanding Gas Barrier Properties, International journal of molecular sciences 20(9) (2019) 2187.

[8] M. Soccio, M. Costa, N. Lotti, M. Gazzano, V. Siracusa, E. Salatelli, P. Manaresi, A. Munari, Novel fully biobased poly(butylene 2,5-furanoate/diglycolate) copolymers containing ether linkages: Structure-property relationships, European Polymer Journal 81 (2016) 397-412. 
[9] G. Guidotti, M. Soccio, M.C. García-Gutiérrez, E. Gutiérrez-Fernández, T.A. Ezquerra, V. Siracusa, A. Munari, N. Lotti, Evidence of a 2D-Ordered Structure in Biobased Poly(pentamethylene furanoate) Responsible for Its Outstanding Barrier and Mechanical Properties, ACS Sustainable Chemistry and Engineering 7(21) (2019) 17863-17871.

[10] G. Guidotti, M. Soccio, N. Lotti, V. Siracusa, M. Gazzano, A. Munari, New multi-block copolyester of 2,5-furandicarboxylic acid containing PEG-like sequences to form flexible and degradable films for sustainable packaging, Polymer Degradation and Stability 169 (2019) 108963-108963.

[11] G. Papamokos, T. Dimitriadis, D.N. Bikiaris, G.Z. Papageorgiou, G. Floudas, Chain Conformation, Molecular Dynamics, and Thermal Properties of Poly( $\mathrm{n}$-methylene 2,5furanoates) as a Function of Methylene Unit Sequence Length, Macromolecules 52(17) (2019) 6533-6546.

[12] R. Storbeck, M. Ballauff, Synthesis and properties of polyesters based on 2,5furandicarboxylic acid and 1,4:3,6-dianhydrohexitols, Polymer 34(23) (1993) 5003-5006.

[13] A. Khrouf, S. Boufi, R.E. Gharbi, N.M. Belgacem, A. Gandini, Polyesters bearing furan moieties, Polymer Bulletin 37(5) (1996) 589-596.

[14] S. Gharbi, J.-P. Andreolety, A. Gandini, Polyesters bearing furan moieties: IV. Solution and interfacial polycondensation of 2,2'-bis(5-chloroformyl-2-furyl)propane with various diols and bisphenols, European Polymer Journal 36(3) (2000) 463-472.

[15] A. Gandini, A.J.D. Silvestre, C.P. Neto, A.F. Sousa, M. Gomes, The furan counterpart of poly(ethylene terephthalate): An alternative material based on renewable resources, Journal of Polymer Science Part A: Polymer Chemistry 47(1) (2009) 295-298.

[16] E. de Jong, M.A. Dam, L. Sipos, G.J.M. Gruter, Furandicarboxylic Acid (FDCA), A Versatile Building Block for a Very Interesting Class of Polyesters, Biobased Monomers, Polymers, and Materials, American Chemical Society2012, pp. 1-13.

[17] A.F. Sousa, C. Vilela, A.C. Fonseca, M. Matos, C.S.R. Freire, G.-J.M. Gruter, J.F.J. Coelho, A.J.D. Silvestre, Biobased polyesters and other polymers from 2,5-furandicarboxylic acid: a tribute to furan excellency, Polymer Chemistry 6(33) (2015) 5961-5983.

[18] J. Carlos Morales-Huerta, A. Martínez de Ilarduya, S. Muñoz-Guerra, Poly(alkylene 2,5furandicarboxylate)s (PEF and PBF) by ring opening polymerization, Polymer 87 (2016) 148158.

[19] G.Z. Papageorgiou, D.G. Papageorgiou, Z. Terzopoulou, D.N. Bikiaris, Production of biobased 2,5-furan dicarboxylate polyesters: Recent progress and critical aspects in their synthesis and thermal properties, European Polymer Journal 83 (2016) 202-229.

[20] Z. Terzopoulou, V. Tsanaktsis, M. Nerantzaki, G.Z. Papageorgiou, D.N. Bikiaris, Decomposition mechanism of polyesters based on 2,5-furandicarboxylic acid and aliphatic diols with medium and long chain methylene groups, Polymer Degradation and Stability 132 (2016) 127-136.

[21] V. Tsanaktsis, Z. Terzopoulou, M. Nerantzaki, G.Z. Papageorgiou, D.N. Bikiaris, New poly(pentylene furanoate) and poly(heptylene furanoate) sustainable polyesters from diols with odd methylene groups, Materials Letters 178 (2016) 64-67.

[22] L. Genovese, N. Lotti, V. Siracusa, A. Munari, Poly(Neopentyl Glycol Furanoate): A Member of the Furan-Based Polyester Family with Smart Barrier Performances for Sustainable Food Packaging Applications, Materials 10(9) (2017) 1028.

[23] D. Maniar, Y. Jiang, A.J.J. Woortman, J. van Dijken, K. Loos, Furan-Based Copolyesters from Renewable Resources: Enzymatic Synthesis and Properties, ChemSusChem 12(5) (2019) 990-999.

[24] P.A. Klonos, L. Papadopoulos, D. Tzetzis, A. Kyritsis, G.Z. Papageorgiou, D.N. Bikiaris, Thermal, nanoindentation and dielectric study of nanocomposites based on poly(propylene furanoate) and various inclusions, Materials Today Communications 20 (2019) 100585. 
[25] L. Papadopoulos, P.A. Klonos, D. Tzetzis, G.Z. Papageorgiou, A. Kyritsis, D.N. Bikiaris, Effects of graphene nanoplatelets on crystallization, mechanical performance and molecular dynamics of the renewable poly(propylene furanoate), Polymer 189 (2020) 122172.

[26] S. Michelina, L. Nadia, M. Andrea, R. Esther, M.-T. Daniel E, Wrinkling Poly(trimethylene 2,5-Furanoate) Free-standing Films: Nanostructure Formation and Physical Properties, 2020.

[27] T.P. Russell, Y. Chai, 50th Anniversary Perspective: Putting the Squeeze on Polymers: A Perspective on Polymer Thin Films and Interfaces, Macromolecules 50(12) (2017) 4597-4609. [28] B. Frank, A.P. Gast, T.P. Russell, H.R. Brown, C. Hawker, Polymer Mobility in Thin Films, Macromolecules 29(20) (1996) 6531-6534.

[29] S. Napolitano, S. Capponi, B. Vanroy, Glassy dynamics of soft matter under 1D confinement: How irreversible adsorption affects molecular packing, mobility gradients and orientational polarization in thin films, The European Physical Journal E 36(6) (2013) 61.

[30] W. Zhang, J.F. Douglas, F.W. Starr, Why we need to look beyond the glass transition temperature to characterize the dynamics of thin supported polymer films, Proceedings of the National Academy of Sciences 115(22) (2018) 5641.

[31] D. McKechnie, J. Cree, D. Wadkin-Snaith, K. Johnston, Glass transition temperature of a polymer thin film: Statistical and fitting uncertainties, Polymer (2020) 122433.

[32] D.E. Martínez-Tong, B. Vanroy, M. Wübbenhorst, A. Nogales, S. Napolitano, Crystallization of Poly(l-lactide) Confined in Ultrathin Films: Competition between Finite Size Effects and Irreversible Chain Adsorption, Macromolecules 47(7) (2014) 2354-2360.

[33] R.M. Van Horn, S.Z.D. Cheng, CRYSTALLIZATION IN POLYMER THIN FILMS: MORPHOLOGY AND GROWTH, Polymer Thin Films, WORLD SCIENTIFIC2008, pp. 163-193.

[34] G. Reiter, I. Botiz, L. Graveleau, N. Grozev, K. Albrecht, A. Mourran, M. Möller, Morphologies of Polymer Crystals in Thin Films, in: G. Reiter, G.R. Strobl (Eds.), Progress in Understanding of Polymer Crystallization, Springer Berlin Heidelberg, Berlin, Heidelberg, 2007, pp. 179-200.

[35] G. Reiter, Some unique features of polymer crystallisation, Chemical Society Reviews 43(7) (2014) 2055-2065.

[36] J. Spièce, D.E. Martínez-Tong, M. Sferrazza, A. Nogales, S. Napolitano, Are polymers glassier upon confinement?, Soft Matter 11(31) (2015) 6179-6186.

[37] H. Yin, S. Madkour, A. Schönhals, Glass Transition of Ultra-Thin Polymer Films: A Combination of Relaxation Spectroscopy with Surface Analytics, in: F. Kremer (Ed.), Dynamics in Geometrical Confinement, Springer International Publishing, Cham, 2014, pp. 17-59.

[38] G. Guidotti, M. Soccio, M.C. García-Gutiérrez, T.A. Ezquerra, V. Siracusa, A. Munari, N. Lotti, Submitted to ACS Sust Chem (2020).

[39] M. Soccio, D.E. Martínez-Tong, A. Alegría, A. Munari, N. Lotti, Molecular dynamics of fully biobased poly(butylene 2,5-furanoate) as revealed by broadband dielectric spectroscopy, Polymer 128 (2017) 24-30.

[40] D.E. Martínez-Tong, M. Soccio, B. Robles-Hernández, G. Guidotti, A. Iturrospe, A. Arbe, S. Arrese-Igor, A. Munari, N. Lotti, A. Alegría, Impact of nanostructure development on the molecular dynamics of poly(pentamethylene 2,5-furanoate), Submitted (2020).

[41] P. Markus, D.E. Martínez-Tong, G. Papastavrou, A. Alegria, Effect of environmental humidity on the ionic transport of poly(ethylene oxide) thin films, investigated by local dielectric spectroscopy, Soft Matter 16(13) (2020) 3203-3208.

[42] D. Nečas, P. Klapetek, Gwyddion: an open-source software for SPM data analysis, Open Physics, 2012, p. 181. 
[43] D. Wang, K. Nakajima, F. Liu, S. Shi, T.P. Russell, Nanomechanical Imaging of the Diffusion of Fullerene into Conjugated Polymer, ACS Nano 11(9) (2017) 8660-8667.

[44] D. Wang, T.P. Russell, Advances in Atomic Force Microscopy for Probing Polymer Structure and Properties, Macromolecules 51(1) (2018) 3-24.

[45] X. Wu, S. Shi, Z. Yu, T.P. Russell, D. Wang, AFM nanomechanical mapping and nanothermal analysis reveal enhanced crystallization at the surface of a semicrystalline polymer, Polymer 146 (2018) 188-195.

[46] C. He, S. Shi, X. Wu, T.P. Russell, D. Wang, Atomic Force Microscopy Nanomechanical Mapping Visualizes Interfacial Broadening between Networks Due to Chemical Exchange Reactions, Journal of the American Chemical Society 140(22) (2018) 6793-6796.

[47] R.I. Rodríguez-Beltrán, D.E. Martínez-Tong, A. Reyes-Contreras, S. Paszkiewicz, A. Szymczyk, T.A. Ezquerra, P. Moreno, E. Rebollar, Laterally-resolved mechanical and tribological properties of laser-structured polymer nanocomposites, Polymer 168 (2019) 178184.

[48] E. Limousin, D.E. Martinez-Tong, N. Ballard, J.M. Asua, Cure-Dependent Morphology of Acrylic/Alkyd Hybrid Latex Films via Nanomechanical Mapping, ACS Applied Polymer Materials 1(8) (2019) 2213-2223.

[49] A. El Sachat, J. Spièce, C. Evangeli, A.J. Robson, M. Kreuzer, M.R. Rodríguez-Laguna, E. Chavez, M. Sledzinska, C.M. Sotomayor Torres, O.V. Kolosov, F. Alzina, Nanoscale Mapping of Thermal and Mechanical Properties of Bare and Metal-Covered Self-Assembled Block Copolymer Thin Films, ACS Applied Polymer Materials 2(2) (2020) 487-496.

[50] J.E. Sader, J.W.M. Chon, P. Mulvaney, Calibration of rectangular atomic force microscope cantilevers, Review of Scientific Instruments 70(10) (1999) 3967-3969.

[51] A.C. Fischer-Cripps, Introduction to Contact Mechanics, Springer US2007.

[52] J. Cui, A. Nogales, T.A. Ezquerra, E. Rebollar, Influence of substrate and film thickness on polymer LIPSS formation, Applied Surface Science 394 (2017) 125-131.

[53] I. Martín-Fabiani, E. Rebollar, S. Pérez, D.R. Rueda, M.C. García-Gutiérrez, A. Szymczyk, Z. Roslaniec, M. Castillejo, T.A. Ezquerra, Laser-Induced Periodic Surface Structures Nanofabricated on Poly(trimethylene terephthalate) Spin-Coated Films, Langmuir 28(20) (2012) 7938-7945.

[54] E. Gutiérrez-Fernández, E. Rebollar, J. Cui, T.A. Ezquerra, A. Nogales, Morphology and Ferroelectric Properties of Semiconducting/Ferroelectric Polymer Bilayers, Macromolecules 52(19) (2019) 7396-7402.

[55] S. Askar, C.M. Evans, J.M. Torkelson, Residual stress relaxation and stiffness in spincoated polymer films: Characterization by ellipsometry and fluorescence, Polymer 76 (2015) 113-122.

[56] D.T.W. Toolan, A. Isakova, R. Hodgkinson, N. Reeves-McLaren, O.S. Hammond, K.J. Edler, W.H. Briscoe, T. Arnold, T. Gough, P.D. Topham, J.R. Howse, Insights into the Influence of Solvent Polarity on the Crystallization of Poly(ethylene oxide) Spin-Coated Thin Films via in Situ Grazing Incidence Wide-Angle X-ray Scattering, Macromolecules 49(12) (2016) 4579-4586.

[57] D.E. Martínez-Tong, L.A. Miccio, A. Alegria, Ionic transport in the amorphous phase of semicrystalline polyethylene oxide thin films, Soft Matter 13(33) (2017) 5597-5603.

[58] J.J. Hernández, D.R. Rueda, M.C. García-Gutiérrez, A. Nogales, T.A. Ezquerra, M. Soccio, N. Lotti, A. Munari, Structure and Morphology of Thin Films of Linear Aliphatic Polyesters Prepared by Spin-Coating, Langmuir 26(13) (2010) 10731-10737.

[59] C.F. Araujo, M.M. Nolasco, P.J.A. Ribeiro-Claro, S. Rudić, A.J.D. Silvestre, P.D. Vaz, A.F. Sousa, Inside PEF: Chain Conformation and Dynamics in Crystalline and Amorphous Domains, Macromolecules 51(9) (2018) 3515-3526. 
[60] G.Z. Papageorgiou, V. Tsanaktsis, D.G. Papageorgiou, K. Chrissafis, S. Exarhopoulos, D.N. Bikiaris, Furan-based polyesters from renewable resources: Crystallization and thermal degradation behavior of poly(hexamethylene 2,5-furan-dicarboxylate), European Polymer Journal 67 (2015) 383-396.

[61] G.Z. Papageorgiou, V. Tsanaktsis, D.G. Papageorgiou, S. Exarhopoulos, M. Papageorgiou, D.N. Bikiaris, Evaluation of polyesters from renewable resources as alternatives to the current fossil-based polymers. Phase transitions of poly(butylene 2,5-furandicarboxylate), Polymer 55(16) (2014) 3846-3858.

[62] T.D. Deshpande, Y.R.G. Singh, S. Patil, Y.M. Joshi, A. Sharma, To study surface and sub-surface nanomechanical properties of electrospun polyacrylonitrile (PAN) nanofibers/polydimethylsiloxane (PDMS) composites, Soft Matter 14(38) (2018) 7829-7838. [63] T. Igarashi, S. Fujinami, T. Nishi, N. Asao, Nakajima, Ken, Nanorheological Mapping of Rubbers by Atomic Force Microscopy, Macromolecules 46(5) (2013) 1916-1922. 


\section{SUPPORTING INFORMATION}

\section{Poly(alkylene 2,5-furanoate)s thin films: \\ morphology, crystallinity and nanomechanical properties}

Beatriz Robles-Hernández ${ }^{1,2}$, Michelina Soccio ${ }^{3} *$, Iker Castrillo $^{2}$, Giulia Guidotti ${ }^{3}$, Nadia Lotti $^{3}$, Ángel Alegría ${ }^{1,2}$, Daniel E. Martínez-Tong ${ }^{1,2, *}$

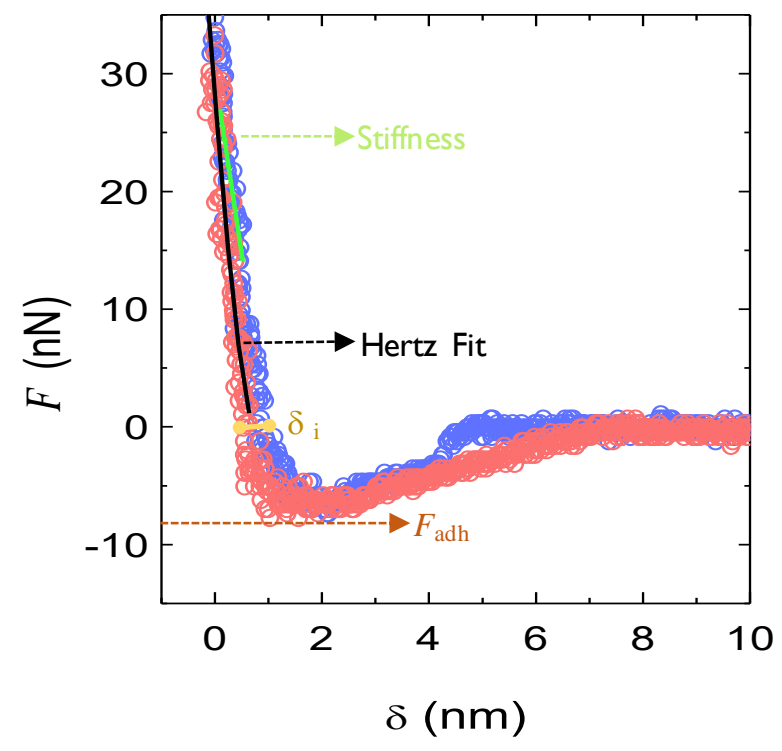

\begin{tabular}{|c|c|c|c|c|}
\hline Stiffness & $\boldsymbol{\delta}_{\mathbf{i}}$ & $\boldsymbol{E}_{\mathbf{Y}}$ & $\boldsymbol{F}_{\text {adh }}$ & $\boldsymbol{R}_{\text {tip }}$ \\
\hline $30 \pm 1 \mathrm{~N} / \mathrm{m}$ & $1.0 \pm 0.2 \mathrm{~nm}$ & $3.3 \mathrm{GPa}$ (fixed) & $7.6 \pm 0.2 \mathrm{nN}$ & $45 \pm 1 \mathrm{~nm}$ \\
& & & & \\
\hline
\end{tabular}

Figure S1. Force spectroscopy measurement and results performed on a PS thin film. 

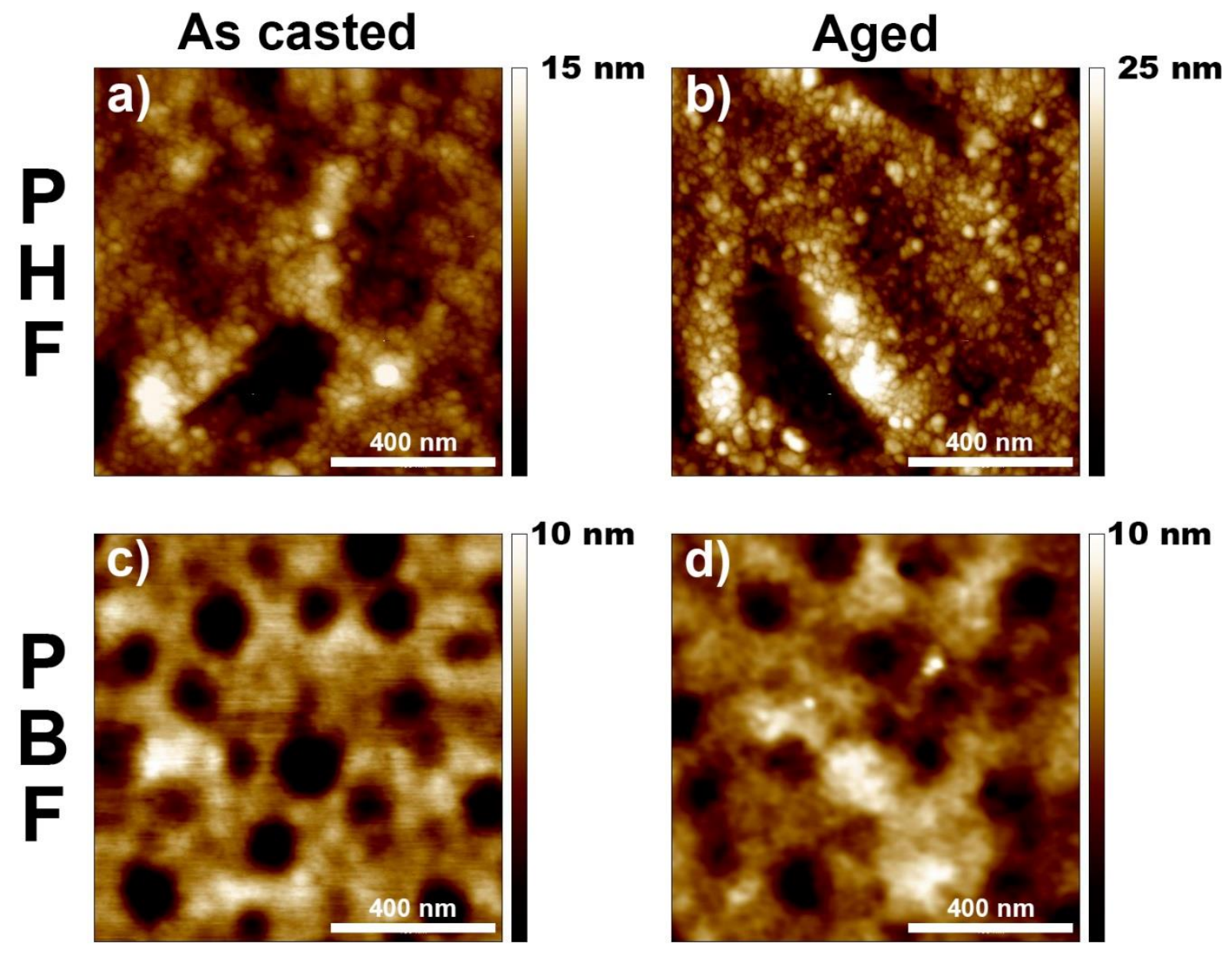

Figure S2. $1 \times 1$ micron AFM height images of PHF (top) and PBF (bottom). Left column corresponds to the as-casted samples, while the right column to the samples after aging for 6 months. 